On the intensity of electronic and vibrational resonant Raman scattering

This content has been downloaded from IOPscience. Please scroll down to see the full text. 1977 J. Phys. C: Solid State Phys. 10 L389

(http://iopscience.iop.org/0022-3719/10/14/004)

View the table of contents for this issue, or go to the journal homepage for more

Download details:

IP Address: 150.163.34.14

This content was downloaded on 25/08/2015 at 19:53

Please note that terms and conditions apply. 


\title{
On the intensity of electronic and vibrational resonant Raman scattering
}

\author{
M A Tenan and L C M Miranda \\ Instituto de Fisica, Universidade Estadual de Campinas, 13100 Campinas SP, Brasil
}

Received 13 May 1977

\begin{abstract}
A discussion of the relative Raman intensities based upon the simple, harmonic single coordinate model for the scattering centre is presented. It is shown that near resonance and for a strong electron-phonon coupling the Raman intensity as a function of the vibrational quantum number $v$ may exhibit a maximum.
\end{abstract}

The theory of the relative Raman intensities in molecules and defects in solids has been the subject of several investigations (Savin 1965, 1966, Verlan 1966, Krushinskii and Shorygin 1961a, 1961b, 1965, Tang and Albrecht 1970, Elliott and Miranda 1975). In all these works the scattering centre was assumed to be represented by a system of at least two electronic energy levels, each possessing vibrational structure, and the molecular wavefunction treated with the Frank-Condon approximation. Based upon this simple model of a scattering centre (molecule) it has been possible to explain some general features of the energy distribution over the components of the scattered spectrum as well as a description of the relation between scattered spectra and electronic absorption spectra. In particular, it might be expected that since the Raman effect uses the electronic polarisability, the electronic excitations would be stronger than the vibrational ones. Experimentally, however, while there are few experimental determinations of absolute Raman intensities (Macfarlane and Ushioda 1970), relative measurements show that the single phonon lines are usually stronger than the electronic ones. Furthermore, recent resonant Raman measurements on $\mathrm{O}_{3}^{-}$centres in $\gamma$-irradiated chlorates and nitrates (Bates and Pigg 1975, Gualberto et al 1977) show an unusual behaviour of the relative vibrational line intensities. In such systems the vibrational Raman intensity is split into two components which are associated with the two non-equivalent positions that the $\mathrm{O}_{3}^{-}$ions can occupy in the lattice. Secondly, for one of these intensity components, the plot of the vibrational Raman intensities as a function of the vibrational quantum number $v$ initially increases, reaches a maximum and then decreases with increasing $v$ (Bates and Pigg 1975, Gualberto et al 1977, Gualberto 1976). As an example, we have reproduced in figure 1 the observed resonant Raman intensities versus $v$ for $\mathrm{O}_{3}^{-}$in $\gamma$-irradiated $\mathrm{Ba}\left(\mathrm{ClO}_{3}\right)_{2} \cdot \mathrm{H}_{2} \mathrm{O}$ (Gualberto et al 1977, Gualberto 1976).

The main motivation of the present Letter has been to try to understand the relative intensity of the various resonant Raman processes depicted in figure 1 within the framework of the previous theories (Savin 1965, Verlan 1966, Krushinskii and Shorygin 1965, Tang and Albrecht 1970, Elliott and Miranda 1975). These theories use the Frank- 


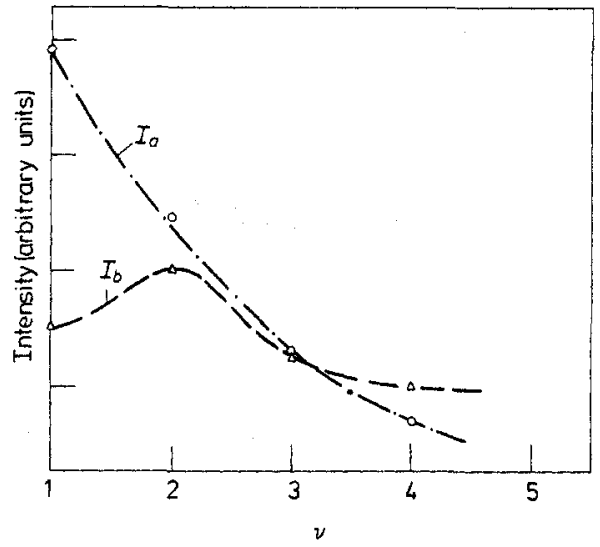

Figure 1. Relative resonant Raman intensities of $\mathrm{O}_{3}^{--}$in $\mathrm{Ba}\left(\mathrm{ClO}_{3}\right)_{2}: \mathrm{H}_{2} \mathrm{O}$ as a function of the vibrational quantum number $v$.

Condon approximation which gives a satisfactory account of optical absorption. Although the simple, harmonic, single coordinate model used is most appropriate for simple molecules it is also reasonable for simple impurities in crystals like F-centres (Kleinman 1964).

The Raman cross section for light scattered from $k_{L}$ to $k_{s}$ can be written as $\sigma=e^{4} \omega_{s} / m^{4} c^{4} \omega_{i}|M|^{2}$ where

$$
M=\sum_{e} \frac{\left\langle f\left|\boldsymbol{\epsilon}_{s} \cdot \boldsymbol{p}\right| e\right\rangle\left\langle e\left|\boldsymbol{\epsilon}_{i} \cdot \boldsymbol{p}\right| i\right\rangle}{E_{e}-E_{i}-\hbar \omega_{L}}+\frac{\left\langle f\left|\boldsymbol{\epsilon}_{i} \cdot \boldsymbol{p}\right| e\right\rangle\left\langle e\left|\boldsymbol{\epsilon}_{s} \cdot \boldsymbol{p}\right| i\right\rangle}{E_{e}-E_{i}-\hbar \omega_{s}} .
$$

Here we assume that the states are separated into an electronic and a vibrational part (Frank-Condon approximation) and that the matrix elements of $p$ are independent of the vibrational coordinates. The latter then enter $M$ only as overlap integrals. If the exciting frequency $\omega_{L}$ is near resonance, the first term in (1) is much larger and we can neglect the second. It is then possible to evaluate the sum over vibrational states in the general case. With these assumptions, a process creating $v$ vibrational quanta has the form :

$$
M=\left\langle f\left|\boldsymbol{\epsilon}_{s}, p\right| e\right\rangle\left\langle e\left|\boldsymbol{\epsilon}_{i} \cdot \boldsymbol{p}\right| i\right\rangle S_{v} / \hbar \omega_{0}^{\prime}
$$

where $\hbar \omega_{0}^{\prime}$ is the vibrational quantum in the upper electronic state and $S_{v}$ takes the simple form

$$
S_{v}=\sum_{n_{e}} \frac{\left\langle 0 \mid n_{e}\right\rangle\left\langle n_{e} \mid v\right\rangle}{n_{e}+\lambda}
$$

In arriving at (3) we have written the energy denominator in units of $\hbar \omega_{0}^{\prime}$. Here $\lambda$ defines the photon matching of the resonance, i.e., $\lambda=\left(E_{e}-E_{0}-\hbar \omega_{L}\right) / \hbar \omega_{0}^{\prime}$. The sum in (3) is over all states $n_{e}$ of the excited oscillator with $\omega_{0}^{\prime}$. The initial and final states are of the ground state oscillator with frequency $\omega_{0}$. The minimum energy of the upper electronic state is at a coordinate position displaced by $r$, say, from the ground state equi- 
librium position so that the peak absorption takes place with the creation of $x=M \omega_{0}^{\prime} r^{2} / 2 \hbar$ phonons. This parameter $x$ represents alternatively a measure of the effective strength of the coupling between the electron and the vibrational mode. The general case of $\omega_{0}^{\prime} \neq \omega_{0}$ has been considered before (Elliott and Miranda 1975), Here we shall consider only the case of $\omega_{0}^{\prime}=\omega_{0}$ which is more appropriate for the experimental situation of Bates and Pigg (1975) and Gualberto et al (1976).

Using the properties of harmonic oscillator functions we find (Elliott and Miranda 1975)

$$
S_{v}=\left(\frac{x^{v}}{v !}\right)^{1 / 2} \frac{\mathrm{d}^{v} S_{0}}{\mathrm{~d} x^{v}}
$$

where

$$
S_{0}=\frac{\mathrm{e}^{-x}}{x^{\lambda}} \int_{0}^{x} \mathrm{~d} t \mathrm{e}^{t} t^{\lambda-1}=\mathrm{e}^{-x} \Gamma(\lambda) \gamma^{*}(\lambda,-x),
$$

$\gamma^{*}(\lambda,-x)$ being the modified incomplete gamma function (Abramowitch and Stegun 1964). Taking the derivatives of $(5), S_{v}$ can be written in a readily calculable form as

$$
\begin{aligned}
S_{v}=\left(\frac{x^{v}}{v !}\right)^{1 / 2} & \left\{\mathrm{e}^{-x} \Gamma(\lambda) \gamma^{*}(\lambda,-x) \sum_{n=0}^{v}\left(\begin{array}{l}
v \\
n
\end{array}\right)(\lambda)_{n} x^{-n}\right. \\
& \left.\quad-\sum_{n=1}^{v}\left(\begin{array}{l}
v \\
n
\end{array}\right) \sum_{m=0}^{n}(-1)^{n-1}(\lambda+m)_{n-m} x^{-(1+n-m)}\right\}
\end{aligned}
$$

where $(a)_{n}=a(a+1) \ldots(a+n-1)$ and $(a)_{0}=1$. The variation of $S_{v}$ with $v$ close to resonance is shown in figures $2(a)-(c)$. It follows from figures $2(a)$ and $(b)$ that near resonance and for $x \gg \lambda$ the $v$-phonon process may become stronger than the $(v-1)$ phonon process whereas for $x$ and $\lambda$ of the same order of magnitude, $S_{v}$ is always smaller that $S_{v-1}$. Furthermore, away from resonance (say $\lambda \gtrsim 1$ ) $S_{v}$ shows the usual behaviour, namely, always decreasing with increasing $v$.

Hence, depending upon the effective electron-phonon coupling (i.e., the value of $x$ ) the resonant vibrational Raman intensity may either present a maximum with respect to $v$ at $v-x$ (strong coupling, $x \gg \lambda$ ) or exhibit a normal behaviour, namely, always decreasing (weak coupling, $x \sim \lambda$ ). This is apparently the case for $\mathrm{O}_{3}^{-}$centres in $\gamma$ irradiated alkali chlorate and nitrates (Bates and Pigg 1975, Gualberto et al 1976) as shown in figure 1 . The two components of the vibrational Raman intensity are associated with the two non-equivalent positions that the $\mathrm{O}_{3}^{-}$ions can occupy in the lattice. In one case (component $b$ of figure 1 ) $\mathrm{O}_{3}^{-}$is more strongly coupled to the lattice than in the other case (component $a$ ). Physically this may be attributed (Bates and Pigg 1975) to the small differences in the static field felt by $\mathrm{O}_{3}^{-}$in the non-equivalent sites occupied by them.

The fact that $S_{v}$ near resonance may exhibit a maximum can be derived from (6) by noticing that for $x \gg \lambda, S_{0}$ may be approximated in lowest order by $x^{-1}$ so that $S_{v}$ then becomes

$$
\frac{(-1)^{v}}{x}\left(\frac{v !}{x^{v}}\right)^{1 / 2}
$$



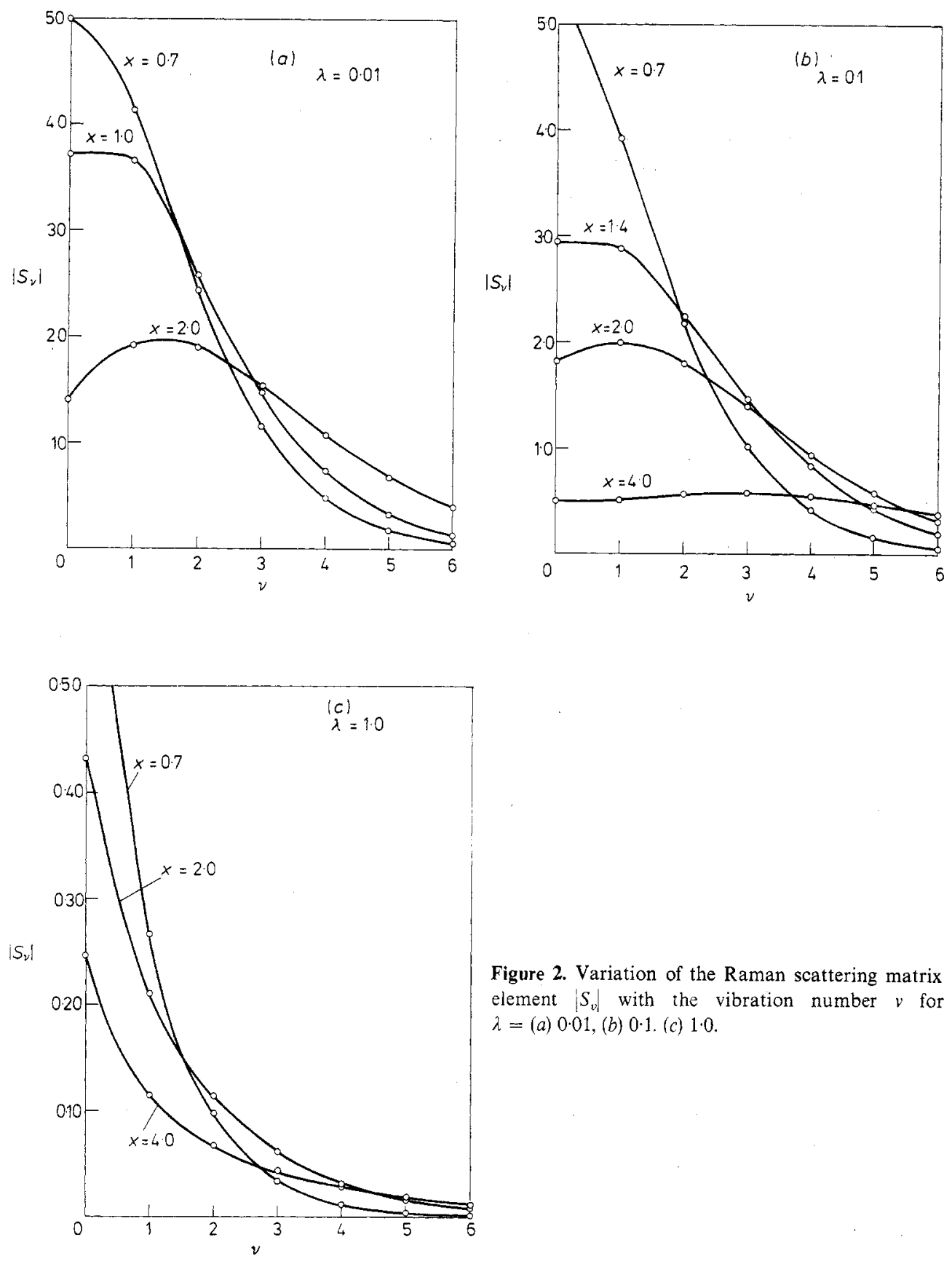

Figure 2. Variation of the Raman scattering matrix element $\left|S_{v}\right|$ with the vibration number $v$ for $\lambda=(a) 0.01$, (b) $0 \cdot 1$. (c) 1.0 . 
Thus for $x \gg \lambda$ one has

$$
\left|\frac{S_{v}}{S_{r-1}}\right| \simeq\left(\frac{y}{x}\right)^{1 / 2}
$$

which means that for $v \gtrsim x, S_{v}$ may become stronger than $S_{v-1}$. Of course there are corrections to (7) which actually prevent $S_{1}$ from growing indefinitely.

Finally, one should also notice that near resonance and for $x \geqslant \lambda$ (see figures $2(a)$ and $(b)$ ) the electronic Raman intensity, which is proportional to $S_{0}$, may be weaker than the first few vibrational overtone intensities. This could possibly be observed near resonance in systems such that $x$ is of the order of unity. Of the existing diatomic molecules whose electronic ground state is a doublet, the best candidates are NO, PO and $\mathrm{ZrO}$ (Herzberg 1950). In the case of NO, $x$ is about 2.0 whereas for PO and $\mathrm{ZrO} x$ is about $0 \cdot 3$ and $1 \cdot 0$, respectively. This therefore means that at resonance the electronic Raman line of NO should be weaker than the one- and two-phonon lines whereas in the case of $\mathrm{PO}$ and $\mathrm{ZrO}$ one should observe the normal behaviour, i.e., the electronic line stronger than the vibrational overtones.

\section{Acknowledgment}

The authors are grateful to Professor R J Elliott for stimulating their interest in this work and for useful discussions.

\section{References}

Abramowitz M and Stegun I A 1964 in Handbook of Mathematical Functions (Washington DC: US GPO) Bates J B and Pigg J C 1975 J. Chem. Phys. 62.4227

Elliott R J and Miranda L C M 1975 in 3rd Int. Conf. on Light Scattering in Solids eds M Balkanski, R C C Leite and S P S Porto (Paris: Flammarion) p 552

Gualberto G M 1976 PhD Thesis Unicamp

Gualberto G M, Tenan M, Vargas H and Miranda L C M 1967 submitted for publication

Herzberg G 1950 Spectra of Diatomic Molecules (New York: Van Nostrand)

Kleinman D A 1964 Phys. Rev. 134 A 423

Kruchinskii L L and Shorygin P P 1961a Opt. Spectrosc. 1112

- 1961b Opt. Spectrosc. 1180

1965 Opt. Spectrosc. 19312

Macfarlane R M and Ushioda S 1970 Solid St. Commun. 81081

Savin F A 1965 Opt. Spectrosc. 19308

1966 Opt. Spectrosc. 20549

Tang J and Albrecht A C 1970 in Raman Spectroscopy vol 2 ed H A Szymanski (New York: Plenum) ch 2 Verlan E M 1966 Opt. Spectrosc. 20341 